\title{
CATALISADORES DERIVADOS DE HIDROTALCITAS APLICADOS NA REFORMA DO BIOGÁS A GÁS DE SÍNTESE
}

\author{
A. V. L. PALADINO ${ }^{1}$ e J.M. ASSAF ${ }^{1}$ \\ ${ }^{1}$ Universidade Federal de São Carlos, Programa de Pós-Graduação em Engenharia Química- \\ PPGEQ-UFSCar \\ E-mail para contato: anandapaladino@gmail.com
}

\begin{abstract}
RESUMO - O aproveitamento do biogás para produção de gás de síntese apresenta um apelo ambiental muito forte, uma vez que são consumidos dois principais gases causadores do efeito estufa: $\mathrm{CH}_{4}$ e $\mathrm{CO}_{2}$. Neste trabalho, foram testados catalisadores derivados de óxidos obtidos a partir de estruturas tipo hidrotalcitas, sintetizadas pela técnica de coprecipitação, contendo níquel como fase ativa, na reação de Reforma Direta do Biogás, bem como na Reforma Oxidativa do Biogás. O tempo de envelhecimento das hidrotalcitas durante a síntese também foi estudado, e tanto os óxidos das estruturas sem e com envelhecimento foram avaliados cataliticamente na Reforma Direta do Biogás, por ser um processo mais severo em comparação à Reforma Oxidativa. Para o conteúdo nominal mássico de $\mathrm{Ni}^{+2}$ correspondente a $5 \%$ no óxido, o catalisador derivado da estrutura não envelhecida se mostrou mais ativo, enquanto que para o conteúdo nominal de $25 \%$ (mássico), o comportamento se inverteu. Razões $\mathrm{H}_{2} / \mathrm{CO}<1$ foram obtidas, ideais para utilização da mistura $\mathrm{H}_{2}+\mathrm{CO}$ na síntese de combustíveis líquidos.
\end{abstract}

\section{INTRODUÇÃO}

Como consequência do aquecimento global e mudanças climáticas dele provenientes, muito esforço tem sido feito para reduzir a emissão dos gases causadores do efeito estufa e incentivar a produção de energias limpas. Ainda referente à preocupação ambiental, sabe-se que o biogás é constituído pelos principais gases responsáveis pelo aquecimento global (aproximadamente 60\% $\mathrm{CH}_{4}$ e $40 \% \mathrm{CO}_{2}$, em volume). Assim, a utilização dessa mistura gasosa numa reação conhecida como Reforma Direta do Biogás, representada pela Reação (1), além de produzir uma mistura conhecida como gás de síntese $\left(\mathrm{CO}+\mathrm{H}_{2}\right)$, a qual pode ser posteriormente utilizada para a síntese de combustíveis líquidos (Fischer-Tropsch) de baixo teor de enxofre e compostos aromáticos, reduz também as emissões dos gases do efeito estufa, em especial pela conversão do $\mathrm{CH}_{4}$. Eventualmente, pode-se adicionar $\mathrm{O}_{2}$ à alimentação dos reagentes, como um agente oxidante extra para eliminação do coque depositado na superfície do catalisador, como representado pela Reação (2) (Reforma Oxidativa) (Lau et al., 2011; Pakhare, Spivey, 2014).

$$
\begin{array}{ll}
0,60 \mathrm{CH}_{4}+0,40 \mathrm{CO}_{2} \leftrightarrow 0,80 \mathrm{H}_{2}+0,80 \mathrm{CO}+0,20 \mathrm{CH}_{4} & \text { Reação (1) } \\
0,60 \mathrm{CH}_{4}+0,40 \mathrm{CO}_{2}+0,15 \mathrm{O}_{2} \leftrightarrow 1,20 \mathrm{H}_{2}+0,90 \mathrm{CO}+0,10 \mathrm{CO}_{2} & \text { Reação (2) }
\end{array}
$$

Uma alternativa para contornar a deposição de coque sobre os catalisadores seria modificar a estrutura dos catalisadores à base de níquel, metal mais utilizado como fase ativa para reações de 
reforma. Catalisadores obtidos a partir do tratamento térmico de estruturas conhecidas como hidrotalcitas podem ser uma solução para esta questão, as quais podem ser obtidas por meio da coprecipitação dos cátions $\mathrm{Ni}^{+2}, \mathrm{Mg}^{+2}$ e $\mathrm{Al}^{+3}$ com um ânion $\mathrm{CO}_{3}^{-2}$, em meio básico, levando à formação de um hidróxido duplo lamelar cujas lamelas são similares ao mineral brucita $\left(\mathrm{Mg}(\mathrm{OH})_{2}\right)$, contendo ânions $\mathrm{CO}_{3}^{-2}$ e moléculas de água no espaço interlamelar. Após tratamento térmico, a hidrotalcita é convertida a um óxido misto precursor de um catalisador que pode ser estável e de boa resistência em relação à formação de coque. Tudo isso devido à formação de uma solução sólida entre o MgO e o $\mathrm{NiO}$ (Tsyganok et al., 2001; Wang et al., 2012).

Neste trabalho, buscou-se estudar o comportamento de catalisador derivado da estrutura hidrotalcita (HT), nas reações de Reforma Direta do Biogás (RDB) e Oxidativa do Biogás (ROB), avaliando-se as conversões dos reagentes a diversas temperaturas de reação, bem como o efeito do envelhecimento da HT na avaliação catalítica.

\section{METODOLOGIA}

\subsection{Sínteses das hidrotalcitas e respectivos óxidos}

As HT's foram sintetizadas por meio da técnica de coprecipitação: uma solução aquosa contendo os nitratos dos metais $\mathrm{Mg}^{+2}, \mathrm{Al}^{+3}$ e $\mathrm{Ni}^{+2}$, tal que a razão $\left(\mathrm{Ni}^{+2}+\mathrm{Mg}^{+2}\right) / \mathrm{Al}^{+3}=3$ e concentração total equivalente a $1 \mathrm{~mol} / \mathrm{L}$, foi adicionada a uma outra solução aquosa contendo $\mathrm{Na}_{2} \mathrm{CO}_{3}$ e $\mathrm{NaOH}$, aquecida a $60^{\circ} \mathrm{C}$, tal que $\mathrm{OH}^{-1} / \mathrm{CO}_{3}^{-2}=11,5$, também a uma concentração total de $1 \mathrm{~mol} / \mathrm{L}$. Durante os 30 primeiros minutos de síntese, o meio reacional foi mantido vigorosamente agitado e, após este período, parte da solução foi retirada para filtração, a fim de separar o gel obtido da água-mãe, enquanto que o restante do meio reacional foi mantido por mais 24 horas a $60^{\circ} \mathrm{C}$, porém sob agitação mais suave, e posteriormente também submetido à filtração. As estruturas obtidas dessas variações, ou seja, sem e com envelhecimento (após 30 minutos e após mais 24 horas, respectivamente), foram filtradas e lavadas com água deionizada a temperatura ambiente, até que o $\mathrm{pH}$ da água de lavagem permanecesse entre 8-9. Os géis resultantes foram então secos em estufa durante 24 horas, a uma temperatura de $80^{\circ} \mathrm{C}$. As HT's sem envelhecimento e com envelhecimento, denominadas $\mathrm{HT}_{0}(\mathrm{NiMg}) \mathrm{AlCO}_{3}{ }^{-2} \mathrm{Ni}^{+2}$ x\% e $\mathrm{HT}_{\mathrm{e}}(\mathrm{NiMg}) \mathrm{AlCO}_{3}{ }^{-2} \mathrm{Ni}^{+2}$ $\mathrm{x} \%$, respectivamente, foram então aquecidas em mufla, da temperatura ambiente até $650^{\circ} \mathrm{C}$, com rampa de aquecimento de $10^{\circ} \mathrm{C} /$ minuto, e mantidas a $650^{\circ} \mathrm{C}$ por 5 horas, obtendo-se aí os óxidos $(\mathrm{NiMg}) \mathrm{AlO}_{\mathrm{x} \% / 0}$ e $(\mathrm{NiMg}) \mathrm{AlO}_{\mathrm{x} \% / \mathrm{e}}$, em que x representa o conteúdo nominal mássico de $\mathrm{Ni}^{+2}$ no óxido final ( $5 \%$ quando a razão molar $\mathrm{Ni}^{+2} / \mathrm{Mg}^{+2}=0,05$ e $25 \%$ quando $\mathrm{Ni}^{+2} / \mathrm{Mg}^{+2}=0,39$ ), enquanto que os índices " 0 " e "e" indicam que os óxidos foram obtidos das $\mathrm{HT}_{0}$ e $\mathrm{HT}_{\mathrm{e}}$, respectivamente. Uma variação do mesmo procedimento descrito anteriormente foi realizado a fim de sintetizar as $\mathrm{HT}_{0} \mathrm{MgAlCO}_{3}^{-2}$ e $\mathrm{HT}_{\mathrm{e}} \mathrm{MgAlCO}_{3}{ }^{-2}$.

\subsection{Caracterização dos precursores}

As HT's e seus respectivos óxidos foram submetidos à análise de Difração de Raios $-\mathrm{X}$, em um equipamento Rigaku Multiflex, usando radiação $\mathrm{K}-\alpha$ de $\mathrm{Cu}(\lambda=1,5406 \AA$, $40 \mathrm{kV}-30 \mathrm{~mA})$, numa varredura de $2 \theta^{\circ}=5-80^{\circ}$, passo de $0,02 \theta^{\circ}$ e velocidade de $2 \theta^{\circ} /$ minuto. As fases presentes nas estruturas foram identificadas através da base de dados JCPDS (Joint Commitee on Powder Diffraction Standards, 1994).

O tamanho do cristalito dos óxidos $\left(\mathrm{L}_{\mathrm{ox}}\right)$ foi calculado a partir da Equação de Scherrer, a 
partir do plano (200), enquanto que a distância entre os cátions na célula unitária ("a") dos óxidos foi calculada a partir da relação $a=2 \cdot d_{(200)}$.

\subsection{Ensaios catalíticos}

Os ensaios catalíticos foram realizados num reator de quartzo amorfo do tipo leito fixo. Para isso, $100 \mathrm{mg}$ dos óxidos foram utilizados para os testes, os quais foram suportados em lã de quartzo no interior do reator. Antes do início dos ensaios, os óxidos foram reduzidos in situ, numa atmosfera de $\mathrm{H}_{2} / \mathrm{N}_{2}(10 / 35 \mathrm{~mL} /$ minuto), a partir de aquecimento da temperatura ambiente até $750^{\circ} \mathrm{C}$ (temperatura definida a partir de ensaios de Redução a Temperatura Programada, não mostrados neste trabalho), numa taxa de $10^{\circ} \mathrm{C} /$ minuto, e mantidos nesta temperatura por 1 hora. Tanto a RDB quanto a ROB foram realizadas a partir de varredura de temperatura, iniciando-se de $800^{\circ} \mathrm{C}$ até $500^{\circ} \mathrm{C}$, com taxa de resfriamento de $10^{\circ} \mathrm{C} /$ minuto. Em ambas as reações, empregou-se razão de $\mathrm{CH}_{4} / \mathrm{CO}_{2}=1,5$, representando a composição típica do biogás, livre de compostos contento enxofre. $\mathrm{Na} \mathrm{RDB}$, juntamente com o $\mathrm{CH}_{4}$ e $\mathrm{CO}_{2}$, alimentou-se $\mathrm{N}_{2}$, tal que a vazão específica fosse $37200 \mathrm{~mL} \cdot \mathrm{h}^{-1} \cdot \mathrm{g}^{-1}$. Na ROB, alimentou-se ar $\left(20 \% \mathrm{O}_{2}\right.$ e $\left.80 \% \mathrm{~N}_{2}\right)$, com razão $\mathrm{O}_{2} / \mathrm{CH}_{4}=0,16$, abaixo do que é requerido estequiometricamente no processo, tal que a vazão específica também fosse $37200 \mathrm{~mL} \cdot \mathrm{h}^{-1} \cdot \mathrm{g}^{-1}$. Os gases efluentes do reator foram analisados num cromatógrafo Varian 450GC, equipado com duas colunas Porapak-N e uma peneira 13X.

\section{RESULTADOS E DISCUSSÕES}

\subsection{Estrutura das hidrotalcitas e dos óxidos precursores dos catalisadores}

Todos os precursores obtidos sem ou com envelhecimento apresentaram difratogramas típicos de HT's: picos agudos e simétricos associados aos planos (003), (006), (110) e (113), e picos mais assimétricos, e de bases mais largas, associados aos planos $(009) /(012),(015)$ e $(018)$, conforme exibidos nas Figuras 1a e 1b (Bîrjega et al., 2005).
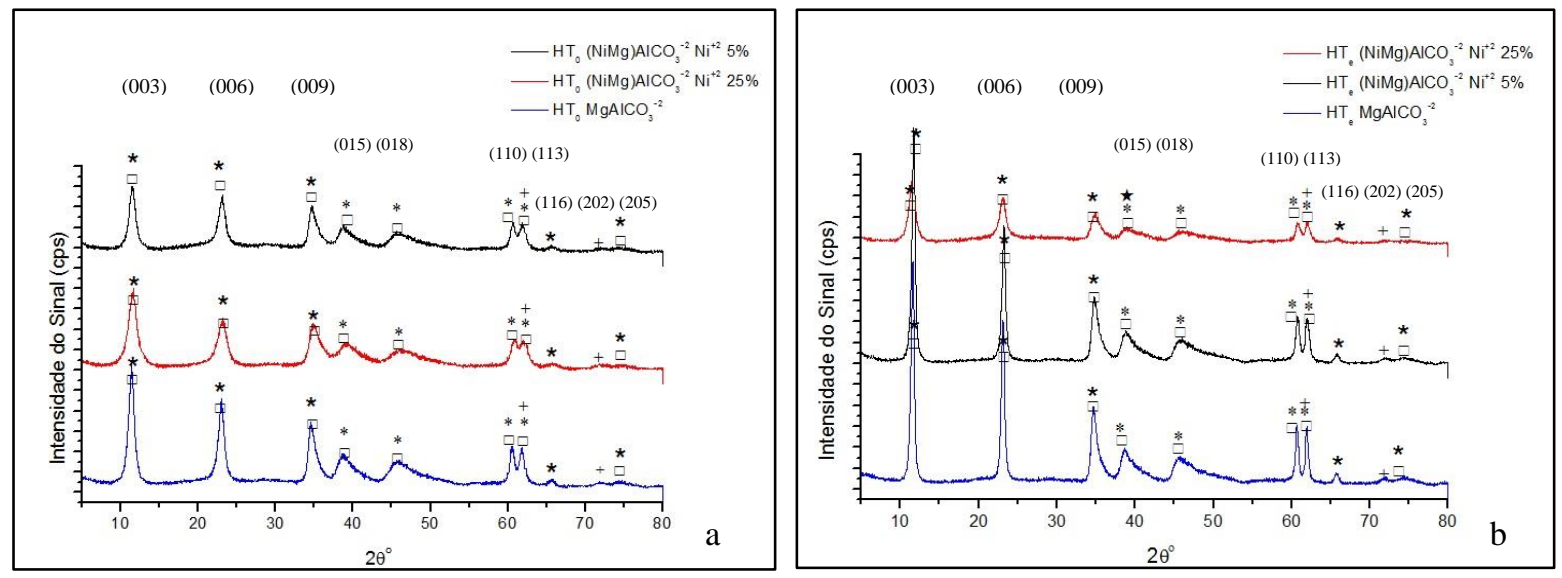

Figura 1a- Difratograma das HT's obtidas após 30 minutos de envelhecimento; Figura 1bDifratograma das HT's obtidas após mais 24 horas de envelhecimento. ( $\square$ ) Hidrotalcita (JCPDS14-0191); ( *) Hidroxicarbonato de Magnésio e Alumínio (JCPDS-70-2151); (*) Hidrargilita $\mathrm{Al}(\mathrm{OH})_{3}$ (JCPDS-02-0215); ( $\left(^{+}\right)$Brucita $\mathrm{Mg}(\mathrm{OH})_{2}$ (01-1169); ( $) \mathrm{Ni}(\mathrm{OH})_{2}$ (JCPDS-01-1047). 
Nota-se que a fase hidroxicarbonato de magnésio e alumínio aparece juntamente com a fase HT, em todos os difratogramas. Nos planos (015), (018), (110) e (113), além da HT, é possível observar também a hidrargilita $\left(\mathrm{Al}(\mathrm{OH})_{3}\right)$, responsável por aumentar a intensidade relativa dos picos, se comparada às fase HT pura, sendo obtida a $\mathrm{pH}>12$, devido ao caráter extremamente básico durante a síntese. A brucita, ou $\mathrm{Mg}(\mathrm{OH})_{2}$ é observada nos planos (113) e (202). Um alargamento da base dos picos, à medida que mais $\mathrm{Ni}^{+2}$ é inserido na estrutura também pode ser observado em especial para os planos (003) e (006), e isso influencia diretamente no tamanho do cristalito. A queda na intensidade do sinal para as variações de envelhecimento ocorre à medida que um maior teor de $\mathrm{Ni}^{+2}$ é inserido na estrutura, exceto para a estrutura sem envelhecimento contendo $25 \%$ de $\mathrm{Ni}^{+2}$ no óxido teórico, cujos sinais se mostraram mais intensos que as de $5 \%$ de $\mathrm{Ni}^{+2}$.

O envelhecimento também leva a um crescimento do cristalito, para todas as estruturas, o que de fato já é esperado. Isto é evidenciado pelo estreitamento da base dos picos dos difratogramas, bem como pelo aumento da intensidade do sinal. Este último fato só não foi observado para a HT contendo o maior teor de $\mathrm{Ni}^{+2}$, muito provavelmente devido à elevada quantidade deste cátion.

As Figuras 2a e 2b mostram a formação dos óxidos mistos pelo colapso das estruturas lamelares das HT's sem e com envelhecimento, respectivamente, com o tratamento térmico destes materiais por 5 horas a $650^{\circ} \mathrm{C}$, pois de acordo com a literatura, estas condições podem levar à formação de uma solução sólida homogênea, em que o cátion $\mathrm{Ni}^{+2}$ se encontra uniformemente disperso na rede do $\mathrm{MgO}$ (González et al., 2013).
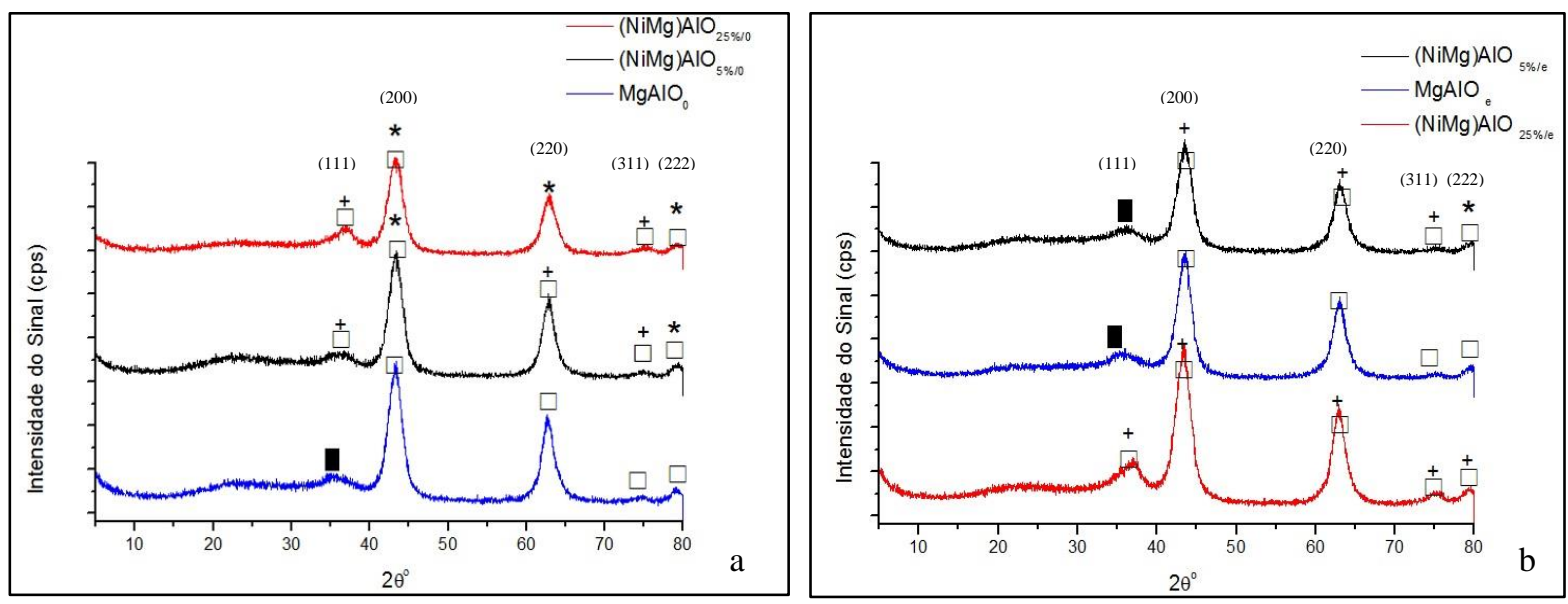

Figura 2a- Difratograma dos óxidos das HT's sem envelhecimento; Figura 2b- Difratograma dos óxidos das HT's com envelhecimento. (口) MgO (JCPDS-75-1525); ( *) NiO (JCPDS-78-0643); $\left(^{+}\right) \mathrm{NiMgO}_{2}$ ( JCPDS-24-0712); (ロ) $\mathrm{MgAl}_{2} \mathrm{O}_{4}$ (JCPDS-03-0897).

Além disso, a partir de ensaios de estabilidade térmica das HT's (não mostrados), não se observou variação significativa das massas das amostras a partir de $600^{\circ} \mathrm{C}$, indicando ausência de transformações químicas a partir desta temperatura.

Para os óxidos obtidos das HT's sem envelhecimento, a inserção do cátion $\mathrm{Ni}^{+2}$ no periclásio 
sob a forma de $\mathrm{NiO}$ ( $\mathrm{a}_{\mathrm{NiO}}$ puro=4,176 ̊ - JCPDS- 24-0712) leva à contração do parâmetro "a" de rede, em relação ao óxido $\mathrm{MgAlO}_{0}$, como pode ser visto na Tabela 1. A contração da rede do periclásio é refletida no deslocamento dos picos do difratograma, como visto na Figura 2a, para ângulos maiores, em relação aos picos do óxido $\mathrm{MgAlO}_{0}$. Já para os óxidos derivados das HT's envelhecidas, observou-se que o $\mathrm{Ni}^{+2}$ se insere na estrutura sob a forma de $\mathrm{NiMgO}_{2}$, fase que está levemente deslocada para a esquerda em relação ao $\mathrm{MgO}$ puro $\left(\mathrm{a}_{\mathrm{NiMgO} 2}\right.$ puro $=4,193 \AA$-JCPDS 24-0712; $\mathrm{a}_{\mathrm{MgO}}$ puro= 4,190 $\AA$-JCPDS-75-1525). Observa-se contração do parâmetro "a" da rede, com a inserção de $\mathrm{Ni}^{+2}$, pelo fato do raio iônico deste cátion ser levemente menor que o do $\mathrm{Mg}^{+2}$ $\left(\mathrm{r}_{\mathrm{Ni+2}}=0,70 \AA, \mathrm{r}_{\mathrm{Mg}+2}=0,72 \AA\right)$. Entretanto, a redução deste parâmetro em relação ao $\mathrm{MgAlO}$ e não é tão acentuada se comparada com a redução dos óxidos da HT não envelhecida, provavelmente pelo fato do $\mathrm{Ni}^{+2}$ se inserir sob a forma de $\mathrm{NiMgO}_{2}$, como já comentado. Ainda em relação aos óxidos da estrutura envelhecida, nota-se uma expansão deste mesmo parâmetro, ao aumentar o teor mássico de $5 \%$ de $\mathrm{Ni}^{+2}$ para $25 \%$, diferentemente do que ocorre com os óxidos derivados da HT não envelhecida. Isso pode ser justificado pelo elevado teor de cátions $\mathrm{Ni}^{+2}$ no óxido $(\mathrm{NiMg}) \mathrm{AlO}_{25 \% / \mathrm{e}}$, os quais não se inseriram fortemente na rede do periclásio.

Tabela 1- Parâmetros cristalográficos dos óxidos

\begin{tabular}{|c|c|c|c|}
\hline Estrutura & $\begin{array}{c}2 \theta^{\circ} \\
(200)\end{array}$ & $\begin{array}{c}\mathrm{a} \\
(\AA)\end{array}$ & $\begin{array}{c}\mathrm{L}_{\text {ox }} \\
(\mathrm{nm})\end{array}$ \\
\hline $\mathrm{MgAlO}_{0}$ & 43,24 & 4,184 & 4,5 \\
\hline $\mathrm{MgAlO}_{\mathrm{e}}$ & 43,52 & 4,158 & 4,7 \\
\hline$(\mathrm{NiMg}) \mathrm{AlO}_{5 \% / 0}$ & 43,30 & 4,177 & 4,5 \\
\hline$(\mathrm{NiMg}) \mathrm{AlO}_{5 \% / \mathrm{e}}$ & 43,58 & 4,153 & 4,7 \\
\hline$(\mathrm{NiMg}) \mathrm{AlO}_{25 \% / 0}$ & 43,38 & 4,171 & 4,2 \\
\hline$(\mathrm{NiMg}) \mathrm{AlO}_{25 \% / \mathrm{e}}$ & 43,52 & 4,158 & 4,2 \\
\hline
\end{tabular}

\subsection{Ensaios catalíticos}

Observa-se na Figura 3a que para os catalisadores ativados a partir dos óxidos (NiMg) $\mathrm{AlO}_{5 \% / 0}$ e (NiMg)AlO $5 \%$, a conversão do $\mathrm{CO}_{2}$ foi sempre superior à de $\mathrm{CH}_{4}$ para a $\mathrm{RDB}$.
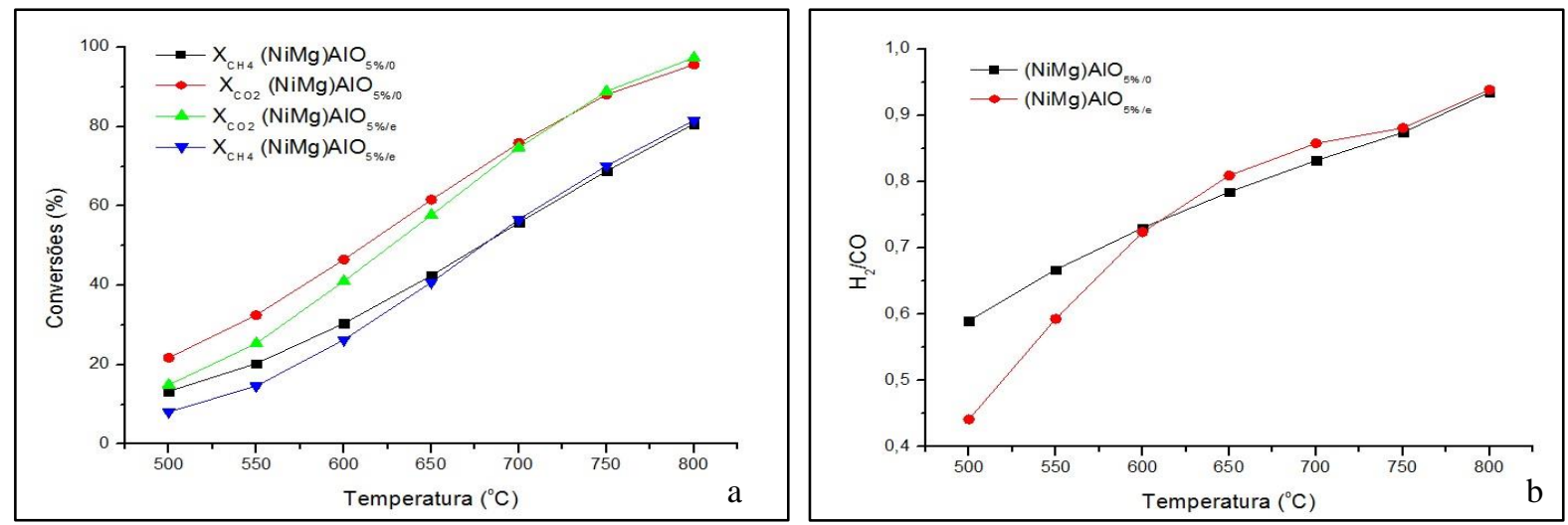

Figura 3a- Conversões de $\mathrm{CH}_{4}$ e $\mathrm{CO}_{2}$; Figura 3b- Razão de $\mathrm{H}_{2} / \mathrm{CO}$ (RDB). 
Primeiramente, segundo Pakhare e Spivey (2014), a barreira de energia para a ativação do $\mathrm{CH}_{4}$ é maior que a do $\mathrm{CO}_{2}$. Essa menor conversão pode ainda ser relacionada com o fato do $\mathrm{CH}_{4}$ estar em excesso em relação ao $\mathrm{CO}_{2}$.

À temperatura de $800^{\circ} \mathrm{C}$, observam-se razões de $\mathrm{H}_{2} / \mathrm{CO}$ próximas a 1 (Figura 3b), porém um pouco abaixo deste valor. Isto acontece uma vez que além da reação de reforma seca do $\mathrm{CH}_{4}$, representada pela Reação (3), ocorre também a reação reversa de deslocamento gás-água (reverse water-gas shift) (Reação (4)) (Asencios, 2013; Lucrédio et al., 2014).

$$
\begin{array}{ll}
\mathrm{CH}_{4}+\mathrm{CO}_{2} \leftrightarrow 2 \mathrm{CO}+2 \mathrm{H}_{2} & \Delta \mathrm{H}^{\mathrm{o}}{ }_{298 \mathrm{~K}}=+247,0 \mathrm{~kJ} / \mathrm{mol} \\
\mathrm{CO}_{2}+\mathrm{H}_{2} \leftrightarrow \mathrm{CO}+\mathrm{H}_{2} \mathrm{O} & \Delta \mathrm{H}^{\mathrm{o}}{ }_{298 \mathrm{~K}}=+41,0 \mathrm{~kJ} / \mathrm{mol}
\end{array}
$$

Observa-se, da Figura $3 b$, que para o catalisador cujo precursor era $(\mathrm{NiMg}) \mathrm{AlO}_{5 \% / e}$ a razão $\mathrm{H}_{2} / \mathrm{CO}$ foi superior em relação à não envelhecida, na faixa de temperaturas entre 600 e $750^{\circ} \mathrm{C}$. Isso pode ser justificado por duas hipóteses: ocorrência da reação de Boudoard (Reação (5)), se a partícula de $\mathrm{Ni}^{0}$ para esta estrutura for maior e, portanto, mais sujeita à decomposição do $\mathrm{CO}$, ou pelo fato da superfície deste catalisador ser menos básica, dificultando a adsorção do $\mathrm{CO}_{2}$. A adsorção de $\mathrm{CO}_{2}$, segundo Pakhare e Spivey (2014), é mais dependente da superfície do suporte do que da quantidade de fase ativa. De qualquer forma, ambas as hipóteses justificam uma menor conversão de $\mathrm{CO}_{2}$ sobre este catalisador, ao longo de todo o experimento.

$$
2 \mathrm{CO}+\mathrm{Ni}^{0} \leftrightarrow \mathrm{C}-\mathrm{Ni}^{0}+\mathrm{CO}_{2} \Delta \mathrm{H}_{298 \mathrm{~K}}^{\mathrm{o}}=-171 \mathrm{~kJ} / \mathrm{mol}
$$

Com o catalisador derivado de $(\mathrm{NiMg}) \mathrm{AlO}_{5 \% / 0}$, a conversão do $\mathrm{CH}_{4}$ foi notadamente superior à do $(\mathrm{NiMg}) \mathrm{AlO}_{5 \% / \mathrm{e}}$, especialmente em temperaturas mais baixas, o que também explica a maior razão $\mathrm{H}_{2} / \mathrm{CO}$ em relação ao $(\mathrm{NiMg}) \mathrm{AlO}_{5 \% / e}$ nesta faixa de temperaturas. Isso pode ser um indício de uma menor quantidade de sítios responsáveis pela adsorção do $\mathrm{CH}_{4}$ no catalisador $(\mathrm{NiMg}) \mathrm{AlO}_{5 \% / \mathrm{e}}$, produzindo uma menor quantidade de $\mathrm{H}_{2}$ a partir da Reação (6). Essa menor quantidade de sítios pode estar relacionada à forma como o $\mathrm{Ni}^{+2}$ foi inserido no óxido da estrutura envelhecida, ou seja, a inserção sob a forma de $\mathrm{NiMgO}_{2}$ tornou mais difícil a exposição do $\mathrm{Ni}^{0}$ na superfície do catalisador.

$$
\mathrm{CH}_{4}+\mathrm{Ni}^{0} \leftrightarrow \mathrm{C}-\mathrm{Ni} i^{0}+2 \mathrm{H}_{2} \quad \Delta \mathrm{H}^{\mathrm{o}}{ }_{298 \mathrm{~K}}=+74,9 \mathrm{~kJ} / \mathrm{mol}
$$

Após ativação do óxido ( $\mathrm{NiMg}) \mathrm{AlO}_{25 \% / 0}$ observou-se, logo no início da varredura, a $800^{\circ} \mathrm{C}$, aumento de pressão ao longo do leito catalítico. Isso pode estar relacionado à sinterização de partículas do catalisador ou, mais provavelmente, à formação de depósitos de material carbonáceo. Devido a isso o teste foi interrompido, uma vez que em menores temperaturas a tendência à formação de coque aumenta, em especial pela ocorrência da Reação de Boudoard (Reação (5)). A impossibilidade de continuidade da reação pode ser justificada pela própria estrutura do óxido precursor, que continha um alto teor de $\mathrm{Ni}^{+2}$, segregado em parte na forma de $\mathrm{NiO}$ no plano (220), como visto na análise DRX. A redução do $\mathrm{Ni}^{+2}$ segregado possivelmente expôs a fase ativa à coqueificação. Por outro lado, o catalisador $(\mathrm{NiMg}) \mathrm{AlO}_{25 \% / \mathrm{e}}$ se mostrou estável ao longo do processo de avaliação catalítica, mostrando que, por mais que houvesse um alto teor de $\mathrm{Ni}^{+2}$ no catalisador, este se inseriu melhor na rede do periclásio, não se tornando tão sujeito à coqueificação, como ocorreu com a estrutura anterior. As Figuras $4 \mathrm{a}$ e $4 \mathrm{~b}$ mostram um 
comparativo das conversões de $\mathrm{CH}_{4}$ e $\mathrm{CO}_{2}$, bem como as razões $\mathrm{H}_{2} / \mathrm{CO}$ entre os catalisadores derivados do $(\mathrm{NiMg}) \mathrm{AlO}_{5 \% / 0}$ e do $(\mathrm{NiMg}) \mathrm{AlO}_{25 \% / \mathrm{e}}$.
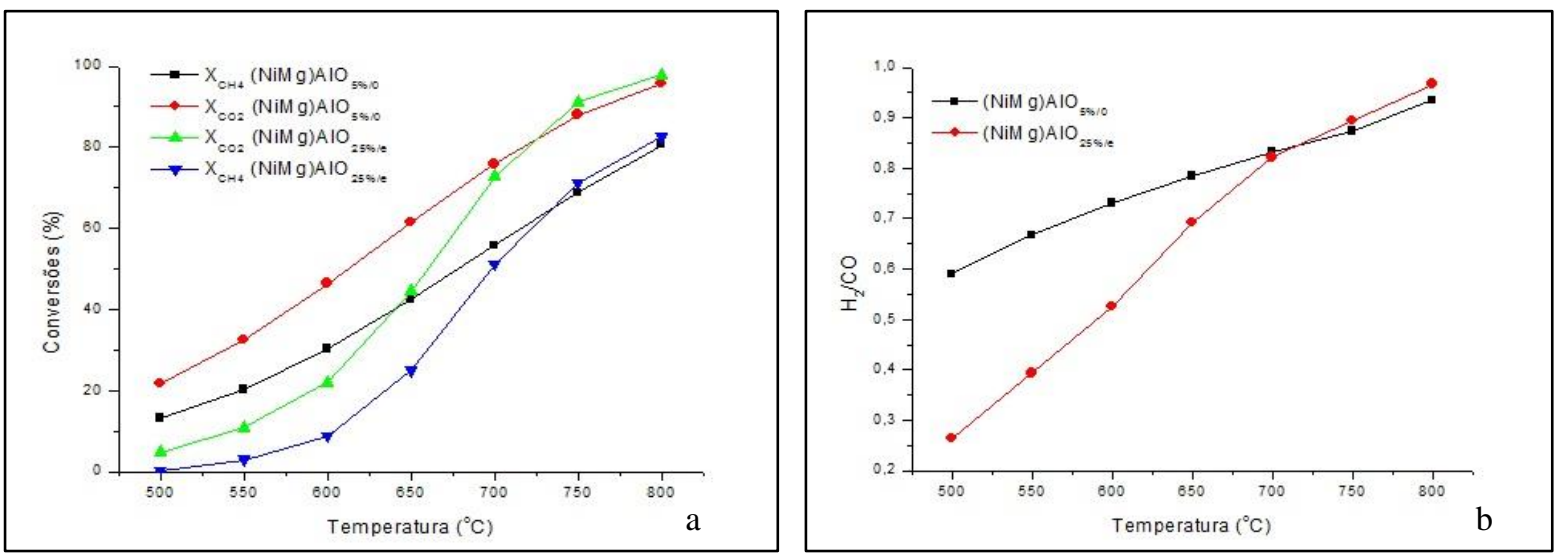

Figura 4a- Conversões de $\mathrm{CH}_{4}$ e $\mathrm{CO}_{2}$; Figura 4b- Razão de $\mathrm{H}_{2} / \mathrm{CO}$ (RDB).

Na Figura 4a, observa-se, acima de $750^{\circ} \mathrm{C}$, conversões de $\mathrm{CH}_{4}$ e $\mathrm{CO}_{2}$ e razões de $\mathrm{H}_{2} / \mathrm{CO}$ similares para ambos os catalisadores. Abaixo desta temperatura, as conversões de $\mathrm{CH}_{4}$ sobre o catalisador derivado de $(\mathrm{NiMg}) \mathrm{AlO}{ }_{5 \% / 0}$ se tornam maiores, provavelmente devido à maior dispersão de $\mathrm{Ni}^{0}$ na superfície do catalisador. As menores conversões de $\mathrm{CO}_{2}$ obtidas com o $(\mathrm{NiMg}) \mathrm{AlO}_{25 \% / \mathrm{e}}$, devem-se provavelmente à maior ocorrência da Reação de Boudoard (Reação (5)), mostrando que as partículas de $\mathrm{Ni}^{0}$, para este catalisador, podem estar mais expostas à formação de coque se comparada com o conteúdo de $5 \%$.

Fazendo um comparativo entre a $\mathrm{RDB}$ e a $\mathrm{ROB}$, para $(\mathrm{NiMg}) \mathrm{AlO}_{25 \% / \mathrm{e}}$, nota-se, da Figura 5a, que a conversão de $\mathrm{CH}_{4}$ na primeira foi menor que na segunda, ao longo de toda a varredura. Entretanto, aqui se observa que em altas temperaturas a razão $\mathrm{H}_{2} / \mathrm{CO}$ é baixa $(<0,8)$ e inferior à razão $\mathrm{H}_{2} / \mathrm{CO}$ na $\mathrm{RDB}$. Isso indica que em temperaturas mais altas, na $\mathrm{ROB}$, parte do $\mathrm{CH}_{4}$ pode estar sendo totalmente oxidado a $\mathrm{CO}_{2}$ e a vapor d'água. Isso reflete o fato de que a oxidação parcial do $\mathrm{CH}_{4}$ pode estar ocorrendo por mecanismo indireto (Asencios, 2013).
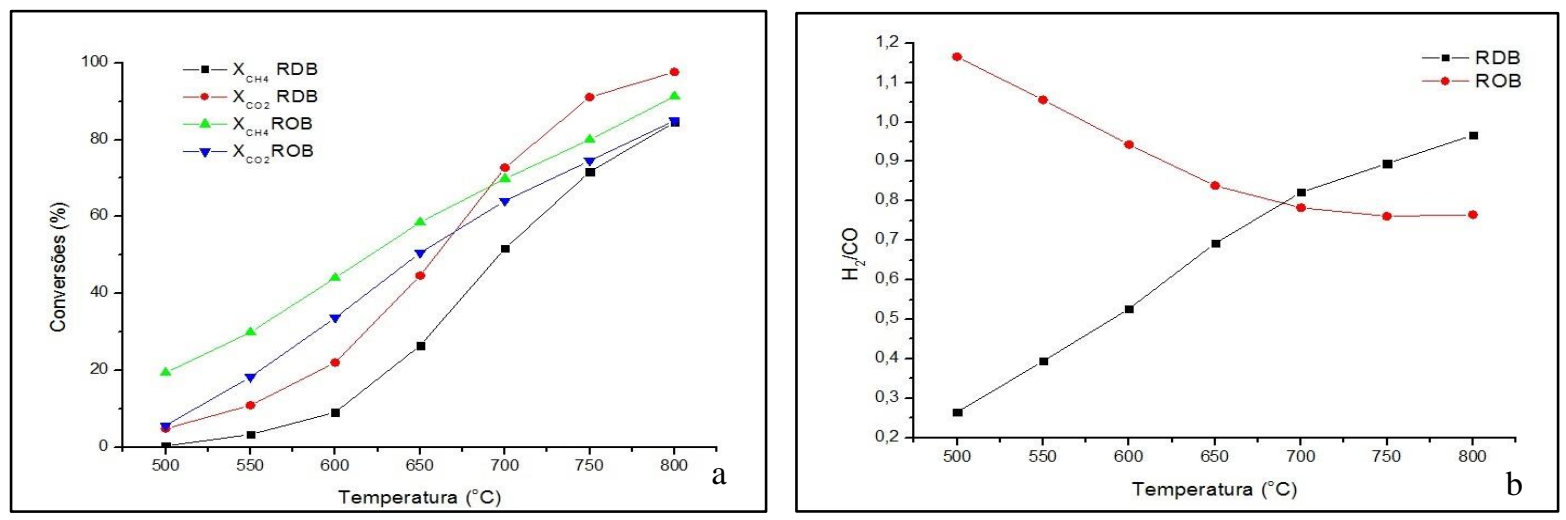

Figura 5a- Conversões de $\mathrm{CH}_{4}$ e $\mathrm{CO}_{2}$; Figura 5b- Razão de $\mathrm{H}_{2} / \mathrm{CO}$ para $(\mathrm{NiMg}) \mathrm{AlO}_{25 \% / e}$. 


\section{Conclusões}

Catalisadores cujos precursores são derivados de óxidos obtidos a partir de HT’s se mostraram ativos para a produção de gás de síntese com razões $\mathrm{H}_{2} / \mathrm{CO}<1$, ideais para sínteses de combustíveis líquidos através de processos do tipo Fischer -Tropsch. Quando o conteúdo nominal mássico de $\mathrm{Ni}^{+2}$ no óxido final foi $5 \%$, o catalisador proveniente da HT não envelhecida se mostrou mais eficiente, tanto em comparação com o derivado do óxido (NiMg)AlO $5 \% / \mathrm{e}$ (em especial a baixas temperaturas), quanto em comparação com o catalisador cujo precursor era o óxido ( $\mathrm{NiMg}) \mathrm{AlO}_{25 \% / \mathrm{e}}$, sendo que este último se apresentou mais estável e ativo se comparado com sua variação não envelhecida. Esta mudança de comportamento com o aumento da quantidade de fase ativa pode estar associada à forma como $\mathrm{o} \mathrm{Ni}^{+2}$ é inserido no óxido com o envelhecimento em combinação com a quantidade deste cátion presente na estrutura. Para 5\% (mássico) de $\mathrm{Ni}^{+2}$, o envelhecimento pode ter dificultado a formação de $\mathrm{Ni}^{0}$ durante a redução do óxido, devido à inserção deste cátion sob a forma $\left(\mathrm{NiMg} \mathrm{O}_{2}\right.$. Para o conteúdo mássico de $25 \%$, entretanto, o excesso de $\mathrm{Ni}^{+2}$ pode ter levado à segregação do mesmo na forma de $\mathrm{NiO}$ no óxido obtido do tratamento térmico da HT não envelhecida, responsável por tornar a fase ativa mais exposta à formação de coque. Uma menor quantidade de $\mathrm{Ni}^{+2}$ no óxido também pode ter gerado um catalisador cujas partículas de $\mathrm{Ni}^{0}$ estejam mais dispersas, aumentando sua atividade catalítica.

\section{Agradecimentos}

Os autores agradecem ao Programa de Recursos Humanos da Agência Nacional do Petróleo, Gás Natural e Biocombustíveis (PRH 44-ANP) pela bolsa concedida e à FAPESP e à CAPES pelo apoio financeiro.

\section{Referências}

ASENCIOS, Y. J. O. Reações de Reforma de Biogás sobre Catalisadores de NiO-MgO- $\mathrm{ZrO}_{2}$ e $\mathrm{NiO}-\mathrm{Y}_{2} \mathrm{O}_{3}-\mathrm{ZrO}_{2} .122$ p. Tese de Doutorado (Doutorado em Química)- Instituto de Química de São Carlos, Universidade de São Paulo, São Carlos. 2013.

BÎRJEGA, R.; PAVEL, O.D.; COSTENTIN, G.; CHE, M.; ANGELESCU, E. Rare-earth elements modified hydrotalcites and corresponding mesoporous mixed oxides as basic solid catalysts. Applied Catalysis A: General, v. 288, p.185-193. 2005.

JOINT COMMITEE ON POWDER DIFFRACTION STANDARDS (JCPDS). USA. 1994.

LAU, C.S.; TSOLAKIS, A.; WYSZYNSKI, M.L. Biogas upgrade to syngas $\left(\mathrm{H}_{2}+\mathrm{CO}\right)$ via dry and oxidative reforming. International Journal of Hydrogen Energy, v. 36, p.397-404, 2011.

PAKHARE, D.; SPIVEY, J. A review of dry $\left(\mathrm{CO}_{2}\right)$ reforming of methane over noble metal catalysts. Chem. Soc. Rev. 2014.

TSYGANOK, A.; SUZUKI, K.; HAMAKAWA, S.; TAKEHIRA, K.; HAYAKAWA, T. Mg-Al Layered double hydroxide intercaleted with $[\mathrm{NI}(\mathrm{EDTA})]^{-2}$ chelate as a precursor for an efficient catalyst of methane reforming with carbon dioxide. Catalysis Letters, v.77, n.1-3, p.75-86. 2001.

WANG, Q.; REN, W.; YUAN, X.; MU, R.; SONG, Z.; WANG, X. Ni catalysts derived from Mg$\mathrm{Al}$ layered double hydroxides for hydrogen production from landfill gas conversion. International Journal of Hydrogen Energy, v. 37, p.11488-11494, 2012. 